\title{
Study on The Theory and Technology of Parallel Training
}

\author{
Zhiming Dong, Qisheng Guo and Yulong Wang* \\ Academy of Army Armored Force, Beijing, China \\ ${ }^{*}$ Corresponding author
}

\begin{abstract}
There are several problems in traditional simulation and live training methods, such as the low technical merit, incoherent process, non-real-time guidance, and subjective assessment and judgement. According to the idea of "real layer semi-physical layer - virtual layer", the basic theory of parallel training for System-of-Systems(SoS) combat is introduced. The basic framework of "one space, two networks and three parallel layers" for parallel training system is constructed. It provides a strong theoretical guidance and effective technical support for the SoS combat of digitized army forces on architecture construction, real-time simulation, real-time guidance, simulation environment flexibility Integration.
\end{abstract}

Keywords-parallel training; virtuality and reality combination; system-of-systems(SoS) combat; LVC

The information warfare is becoming more and more systematic and complicated. The network-centric combat capability has become the concentrated embodiment of the combat effectiveness of troops. Actual and System-of-Systems (SoS) combat training is the fundamental guarantee of the generation and promotion of combat effectiveness. However, the current training methods are mainly a single simulation or live exercises, and most of these training or exercises are SoS combat training which organized by plan and scheduling. Numerous interrupt of guidance staff result in incoherent process, non-real-time guidance, subjective assessment and judgement. It is difficult to meet the needs of the actual SoS combat.

\section{STATUS AT HOME AND ABROAD}

\section{A. TENA Technology}

Test and Training Enabling Architecture (TENA) [1] is the achievement of the Foundation Initiative 2010 (FI2010) project sponsored by US Department of Defense(DoD). It is put forward to promote the interoperability, reusability and composability of test and training resources, the architecture is shown in Figure 1. It can integrate the test, training, simulation, high-performance computing resources distributed in different ranges and facilities to construct a number of test and training "logic range" according to specific tasks. The US army Millennium Challenge 2002(MC02), Integral Fire 07 (IF07), 2009 Joint Expeditionary Force Experiment(JEFX09) and Joint Mission Environmental Test Capability (JMETC) utilize TENA framework, to provide a distributed, real-time, virtual and component test and training capacity. The geographically distributed test and training resources are integrated to foster a virtuality and reality combination training platform.

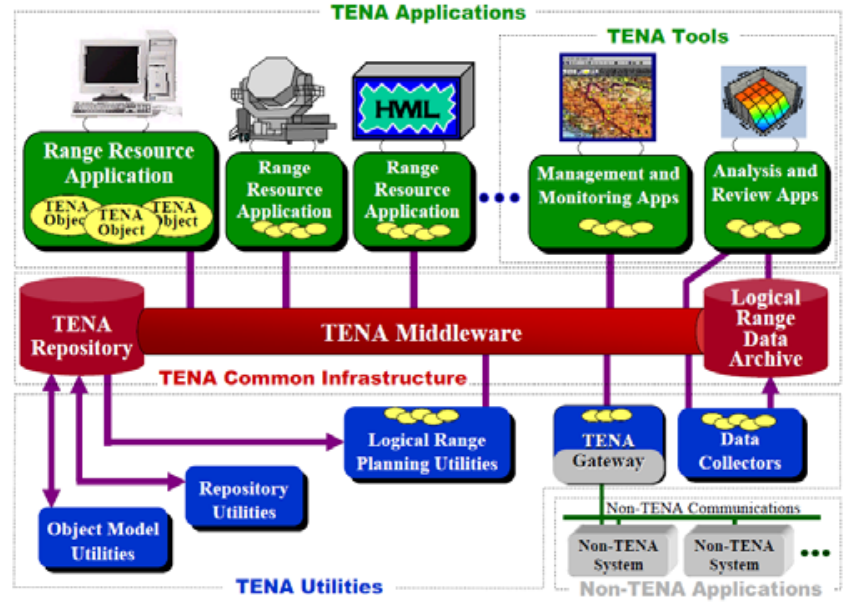

FIGURE I. TENA ARCHITECTURE

\section{B. Parallel System Approach}

In 2004, Wang Fei-yue, professor of the Chinese Academy of Sciences (CAS) Automation Institute, proposed the Artificial System, Computational Experiments, Parallel Execution (ACP) [2], which is a new idea and methodology to solve the problems of complex system modeling, analysis, control and organization management. ACP firstly establishes a virtual complex system through artificial system or artificial social modeling method on computer. Secondly, using complex systems calculation to study and design complex social behavior and action experiment in real world, quantifiably analysis and evaluate the influence factors of complex systems. Finally, utilizing the complex system parallel execution method to synchronously carry forward and supplementally analysis the virtual complex system and actual system, parallelly control and manage their actions, adjust the management and control manner of actual system through the "reference" and "estimation" of their future development and evolution. Then, the integration of complex system computing experiments and parallel implementation is constructed.

\section{US Air Force Red flag Training}

The US Air Force Red Flag exercise is the most practical aerial exercise in the Western world. It is a typical example of the actual combat exercise using virtual reality interaction and the highest form of tactical training for American Air Force tactical forces[5]. The most advanced Red Flag - Alaska (RF-A) exercise is performed by Joint Pacific Alaska Range Complex (JPARC). JPARC uses the Test and Training Enabling Architecture (TANA) as a heterogeneous entity interaction 
mechanism, operates the exercise guidance and control system and virtual battlefield system on the ground and air early warning, accesses various weapon systems that come from different countries, collects weapon systems state data with data acquisition system and transform them to virtual battlefield space through JPARC's broadband data transmission system (microwave, fiber, etc.) in real time. The information generated by different space and ground platforms create a virtual battlefield scene, which provides interaction and damage calculation, impacts the exercise equipments data in real time. During the air combat, "destroyed" aircrafts must leave, so they will not affect the rest of participants.

\section{APPLICATION REQUIREMENTS}

Because of the low technical merit, traditional simulation and real combat methods can hardly afford the needs of the generation and promotion of new quality combat effectiveness. Therefore, using ACP method and learning from the US TENA architecture technology and the test and training experience based on virtuality and reality combination, a virtuality and reality combination, real-time and parallel operation training model can be built. Its main implement requirements include four parts.

\section{A. Access Fusion}

Virtuality and reality combination refers to numerous live (L), semi-physical simulator (V), virtual force (C) training resources. However, each system has different system structure, interaction and promotion mechanism, standard specification, description granularity and simulation step size of the entity and action. Moreover, so many geographically distributed heterogeneous systems have cost a lot of manpower, material and financial resources, and been successful applied in relevant field. The available logic range resources should not be completely overturned. The virtuality and reality combination is able to provide interface or general access means for these systems to achieve seamless connectivity with existing resources.

\section{B. Real-time Interaction}

The platform of training based on man-in-loop and actual equipment-in-the-loop should be real-time. The logical time consistency of interoperability between the two entities should meet the simulation accuracy requirements of the interoperability process. At the same time, the virtuality and reality combination training should not only consider lowspeed equipments (such as cars, tanks, etc.), but also access aircraft, missiles and other high-speed flying weapons. The time-space consistency and real-time interaction of the variety of concomitant range resources should be ensured when highspeed weapons (speed up to 5 Mach or more) join the training and the system elements conduct strong interaction. In general, the delay should meet the man-in-loop and actual equipment in-the-loop training real-time needs.

\section{Flexible Configuration}

Different types of training obtain different requirement about resources. One platform should support both single-skill training and unit, system training. Only flexible configuration environment can achieve the flexible allocation and reuse of resources to meet different needs. The main problems should be solved are as follows, training resources flexible access technology, resource management and scheduling technology, dynamic seat configuration technology, software operation and maintenance technology, fast and reliable system deployment technology.

\section{Application Compatibility}

Virtuality and reality combination training should not only support the training of army based on information systems and other services, but also provide full life cycle tool support, data support and environmental support. Take account of the numerous requirements about simulation model, data extraction and processing of different types of training, multi-resolution model and scale of the cutting may be the key considerations.

\section{Parallel Training TheOry}

\section{A. Basic Concepts}

Parallel training (PT) operates on parallel training system which combines "real layer - semi-physical layer - virtual layer", integrates base training, simulation training and network training, carries out actual SoS combat relying on information technology equipment system.

1) Parallel Training System: Parallel training system builds "real-layer - semi-physical layer - virtual layer" in accordance with ACP theory, utilizes equipment real-time state acquisition, wireless broadband transmission, positioning and virtual battlefield environment, projects the equipment SoS combat state information of "real layer - semi-physical layer" to virtual battlefield space, constructs red and blue engagements combat simulation with "virtual layer", and transforms combat information back to "real layer - semiphysical layer" equipments. Parallel layer and its basic concept are shown in Figure 2.

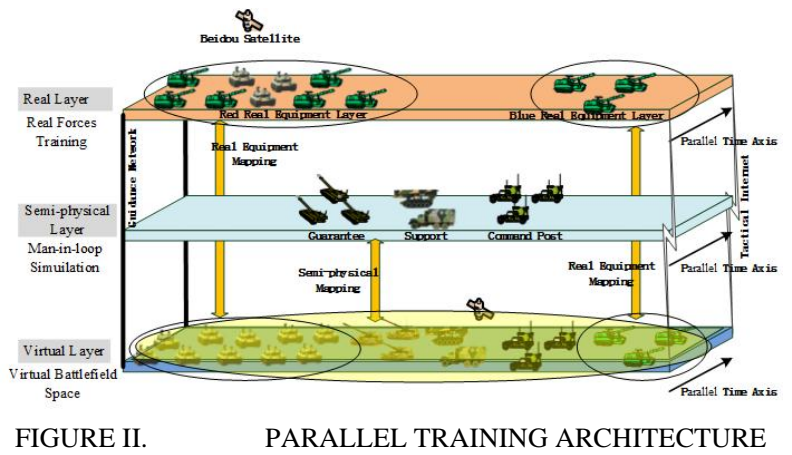

2) Base Training, Simulation Training and Network Training Integration: Parallel training needs typical tactical training ground. The main reason is that during the construction of parallel training system, a virtual battlefield space according to real battlefield space should be established to synchronously offer battlefield environment data foundation for the three parallel layers. Parallel training also needs to construct a wired and wireless combination guidance and control broadband network to ensure data transmission between parallel layers in real time; Parallel training can access the semi-physical simulator and virtual forces as a 
complement for the real system. Therefore, parallel training is a new training model that combines the advantages of base training, simulation training and network training.

3) Information Equipment System and Real SoS Combat: Parallel training is on the basis of information and primary tactical Internet construct capability. A large number of reconnaissance intelligence, electronic warfare, communication and command control simulation (virtual) equipment of semi-physical layer and virtual layer can be used to simulate command posts, supplement other forces, and make up an integrated equipment system with real layer equipments. Virtuality and reality fusion tactical Internet can be built by bridging technology. These semi-physical simulators (virtual equipment) are equipped with real tactical software and related business software, can achieve connectivity with the real layer command and control software. Therefore, tactical Internet interactive information can be displayed in the virtual battlefield space and gathered by guidance and control system in real time, which do a good job for command information system preparation of real SoS combat.

To sum up, parallel training builds a virtual battlefield space in line with the real battlefield space, projects the equipments of real battlefield to the virtual battlefield space in real time, sets up a perfect combat system in virtual battlefield. In virtual battlefield, it is able to deal with actions and status information of fire strikes, equipment damage, performance evaluation, transmit calculation results of SoS combat back to real battlefield, conduct the operations and processes, assess training levels in real time. Therefore, SoS combat training of real forces can be achieved.

\section{B. Overall Structure}

The overall structure of the parallel training system can be summarized by "one space, two networks, three parallel layers", as shown in Fig 2. "one space" refers to the real battlefield and virtual battlefield should be realistic synchronization. The real battlefield, real equipments, semi-physical simulation equipments and virtual forces should be projected to the virtual battlefield, and a virtual combat space which is the core of the parallel training system can be established. "two networks" refers to the guidance and control network and tactical Internet. Guidance and control network is responsible for the connectivity of guidance, real equipment information collection and battlefield display information. Tactical Internet ensures the connectivity and non-interference of combat information. " three parallel layer" refers to the "real layer - semi-physical layer - virtual layer". The actual equipments concentrate in the real layer. All the training personnel concentrate in the real layer and semi-physical layer. Guidance, control and management concentrate in the virtual layer which can generate virtual forces to improve SoS combat effect.

\section{Operation Mode}

Parallel system operation mainly includes three types, such as learning and training, experiment and evaluation, control and management. In parallel training, the real layer and semiphysical layer can be considered as the artificial systems of parallel system, virtual battlefield space can be considered to be calculation experiments, the synchronized and paralleled real battlefield and virtual battlefield can be considered as parallel implementation. Therefore, parallel training system conforms to all the characteristics of parallel system, is able to be calculated and realized with the parallel system operation management mode. Parallel training conducts the effective control, management and command of complex military system based on the real-time and paralleled interaction between actual military system and virtual battlefield space.

In parallel training, equipments and personnel distribute in different battlefield space. Direct aim equipments and personnel are deployed in real layer, indirect aim (or nonvisible) equipments and personnel are generally deployed in the semi-physical layer, other equipments and personnel can be arranged in the virtual layer. Equipments and personnel in the three parallel layers make up the red and blue engagements. The equipments and personnel in different parallel layer have a perfect equipment system, logically in the same time and space in the battlefield, similarly in the same time and battlefield space by feeling. They can be interrelated, matched and antagonistic. They are in the same logical space, their time logic is consistent, but in real space they are paralleled.

\section{KeY TEChNOLOGIES}

\section{A. Parallel Training System Architecture Technology}

The core technical problem of the parallel training system is the interoperability of heterogeneous resources. It is necessary to packaging the training resources such as actual equipments, simulators and mathematical models based on a unified architecture, establish a uniform language and communication mechanisms, build a logical range with a unified time, space and battlefield environment. Connectivity and interoperability technology based on middleware is the core of the architecture. The middleware integrates distributed shared memory encoding extraction, anonymous publish and subscribe, distributed model-driven and object-oriented programming into a middleware system. The core of the architecture is a group of middleware software collection that provides centralized management, data transmission, scheduling services and time services for parallel training. It can achieve the connectivity and interoperability of all kinds of virtual and reality model, training objects and guidance resource without modifying the existing models. The data of publish and subscribe are serialized and then symmetrically encrypted and transferred. The interoperability technology of training resource is the key to the architecture. The architecture object model is defined by meta-model (the model describes other models by defining an abstract language). The meta-model defines some basic concepts, restrictions and rules. The heterogeneous system integration technology based on "gateway + adapter" is an important component of the architecture. The architecture integrates different types of systems through heterogeneous gateways and adapters. The TISA gateway / adapter map heterogeneous systems to object model of the architecture using proxy model mechanism. Gateway / adapter includes tactical Internet gateway, actual equipment adapter, DDS gateway and HLA gateway. 


\section{B. Real-time Simulation Technology Based on Fault- Tolerant Permutation}

The three parallel layer object simulation process of parallel training system may introduce different types of failure. The proxy model of "real object" and "semi-physical object" is essentially a "virtual force object". There are mainly three types of failure. The first type of failure is superframe, real-time system information update superframe caused by communication network restriction. Such failures generally continue only a few simulation cycles, and the data of the relevant frame can be obtained after communication recovery. The second type is recoverable failure, the malfunction due to "real object" adaptor or "semi-physical object" gateway breakdown. Relevant equipments can regain the access by restarting or renewal. This type of failure often subjects to minute delay. The third type is permanent failure, refers to the "real object" and "semi-physical object" occurs serious failure which can not be restored in the entire LVC test and training process.

In order to ensure the real-time performance of parallel training system simulation, three types of failure are classified. Perform fault-tolerance with estimate interpolation method for the first type of failure. The agent model performs cubic spline interpolation based on the previous cached data, extrapolates a frame based on the spline function obtained from interpolation result, and starts counting. When the count value is greater than $\mathrm{N}$, start the second type of fault-tolerance mechanism. $\mathrm{N}$ is the first type of failure threshold which according to the error tolerance of different LVC application scenarios. When the threshold is enabled to start the second type of failure faulttolerance mechanism, the process of the original agent model must be terminated and the original agent model should be replaced with the virtual force model that is isomorphic with the "real object" or "semi-physical object". At this time, manin-loop have been replaced by computer generated force (CGF). At this point, if the failure can be restored, the vehicle crew can make a recovery request to the LVC test operation and control system, go to the designated location to re-initialize by manual scheduling, and finally rejoin the LVC simulation system. If the failure is not recoverable, refers to the third type of failure, the vehicle group will be replaced by a virtual force model from start to finish.

\section{Simulation Environment Flexible Integration Technology}

SoS combat is a complex and large-scale system problems. Digital mechanized division simulation command level can reach 7 to 8 , contains 300 types of equipments. Digital mechanized division simulation is provided with strong system antagonism, complex network structure, more influencing factors. To achieve the simulation of complex system, using fixed simulation method for each equipment and function will introduce poor adaptability when the simulation objects change. Therefore, simulation environment construction needs new ideas and methods. The simulation environment flexible integration technology based on combination of man-in-loop and CGF can adapt to the large-scale and fast changing simulation objects. It realizes on-demand combination and flexible integration mainly based on functional integration, job integration, equipment integration and unit integration. Function integration mainly realizes the combination of hardware equipment function and software function, achieves the rapid deployment of the command information system and replacement of equipment terminal. Job integration is mainly to generate the specific function equipment based on function integration. Equipment integration mainly assembles the different equipment to combat unit according to the requirement of arrangement. Unit integration is the polymerization simulation mainly in virtual force bedding. In order to realize flexible integration, a series of technologies such as virtual desktop, cloud management, semi-physical equipment generalization, electromagnetic environment simulation and link communication layer simulation are achieved, the problems of job function configuration, command and control software deployment, equipment performance simulation and virtual force aggregation are solved, the generalization of simulation environment is obtained.

\section{Real-time Guidance Technology Based on Parallel Control}

At present, the battlefield data collection, emergency situation disposal, combat results assessment, exercise process guiding of actual SoS combat training are mainly relying on the monitoring, observation and on-site disposal of guidance personnel. The guidance, control and evaluation sub-system mainly includes the functions that network transmission, video surveillance, information collection input/output, data statistics and analysis, rarely obtains data collection and situation judgement. This kind of guidance, control and evaluation method involves too much manual participation. Information input by artificial results in the difficulty of the real time combination of system and exercise, poor data accumulation and quality, subjective evaluation. It is difficult to meet the demands of real-time SoS combat.

Real battlefield and virtual battlefield in parallel training system gain synchronized execution and parallel promotion. Middleware achieves the logic battlefield real-time information connectivity. Real-time guidance system accesses equipment and battlefield interactive information from middleware, obtains the parallel domination and real-time guidance of real battlefield, conducts real-time assessment and adjudication and feeds them back to the real training system. Using real-time guidance technology of parallel control and parallel system approach, real-time guidance management integration, data collection, decision making, test and exercise process promotion can be achieved.

\section{CONCLUSION}

The parallel training theory is a milestone for actual combat training. It combines the base training, simulation training and network training. It is a high-level form of real forces SoS combat training, which can meet the requirements of digitized troops actual combat training and has a very important practical significance for the formation and promotion of network centric combat capability. Parallel training and its system can be used in various types of military training base. It can gradually generate combining with the construction of specialized blue army and exercise training site. The parallel training theory can be widely used and greatly improve the army actual combat capability. 


\section{REFERENCES}

[1] The Test and Training Enabling Architecture Reference Document [EB/OL]. www.tena-sda.org/documents/tena2016.pdf, TENA Software Development Activity, 2016.

[2] F. Y. Wang, Artificial society, computational experiment, parallel system - discussion on complex socio-economic system calculation, Complex Systems and Complexity Science, no. 4, pp. 12-20, 2004.

[3] F. Y. Wang, Parallel system methods for management and control of complex systems, Control and Decision, vol. 19, no. 5, pp. 485-489, 2004.

[4] F. Y. Wang, War organization and action for cyberspace: discussion on parallel military systems, Military Operations and Systems Engineering, vol. 26, no. 3, pp. 5-10, 2012.

[5] Red flag, http://wapbaike.baidu.com/view/1692342.htm, Baidu Encyclopedia, 2017.

[6] Y. R. Tang, Z. Li. Research on the Approach of Space System Modeling and Simulation Based on the Parallel System Thought, Journal of Academy of Equipment, vol. 24, no. 4, pp. 67-70, 2013.

[7] Y. Zhang, M. Z. Zhang, X. F. Hu, On Multi-System Integration Technology Oriented to LVC Training, Journal of System Simulation, vol. 25, no. 11, pp. 2515-2521, 2013.

[8] Z. M. Dong, Study on Theory and Methods of Parallel Training for System-of-System Combat, Journal of Academy of Armored Force Engineering, vol. 30, no. 1, pp. 63-68, 2016. 